https://artnodes.uoc.edu

\title{
Estrategias del cine volumétrico para generar empatía en la audiencia. El caso de Queerskins: Ark
}

\author{
Francisco-Julián Martínez-Cano \\ Universidad Miguel Hernández \\ Emilio Roselló-Tormo \\ Universidad Miguel Hernández
}

Fecha de presentación: abril de 2021

Fecha de aceptación: mayo de 2021

Fecha de publicación: julio de 2021

\section{Cita recomendada}

Martínez-Cano, Francisco-Julián; Roselló-Tormo, Emilio. 2021. «Estrategias del cine volumétrico para generar empatía en la audiencia. El caso de Queerskins: Ark». [artículo online]. En: González Díaz, Paloma; García Méndez, Andrea (coord.) «En los límites de lo posible: arte, ciencia y tecnología». Artnodes, no. 28. UOC. [Fecha de consulta: dd/mm/aa]. http://doi.org/10.7238/a.v0i28.38681

\section{Resumen}

El registro volumétrico ofrece la posibilidad de trabajar con grabaciones de actores reales en producciones cinematográficas interactivas de realidad virtual (RV). Queerskins: Ark (Szilak y Tsiboulski, 2020) es una de las primeras creaciones narrativas que aplican y exploran esta técnica, capaz de potenciar las respuestas empáticas de la audiencia. El presente trabajo parte del análisis de esta obra, con el objetivo de identificar los procedimientos de creación empleados en su construcción. Este estudio emplea una metodología cualitativa que se compone de tres fases. Una primera de revisión bibliográfica, para, posteriormente, profundizar en el proceso de creación de Queerskins: Ark a través de una serie de entrevistas semiestructuradas a los 


\title{
artnodes
}

https://artnodes.uoc.edu

Estrategias del cine volumétrico para generar empatía en la audiencia. El caso de Queerskins: Ark

autores. Finalmente se analiza el metraje virtual aplicando un modelo propio diseñado en tres bloques. Los proyectos de investigación, llevados a cabo para comprobar el impacto de la narrativa audiovisual de RV en el espectador, siempre han optado por las estrategias de toma de perspectiva. La obra objeto de estudio, por el contrario, experimenta con la aplicación de recursos expresivos y artísticos con el mismo fin. Los resultados expuestos demuestran la existencia de estrategias alternativas para generar respuestas empáticas en la audiencia.

\section{Palabras clave}

cine volumétrico, efecto presencia, empatía, realidad virtual, producción audiovisual

\section{Volumetric film strategies to generate empathy in the audience. Case study of Queerskins: Ark}

\begin{abstract}
Dolumetric recording offers the possibility of working with recordings of real actors in interactive virtual reality film productions. Queerskins: Ark (Szilak and Tsiboulski, 2020) is one of the first narrative creations to apply and explore this technique, which is capable of enhancing the audience's empathic responses. This paper starts with the analysis of this work, with the aim of identifying the creative procedures used in its construction. This study employs a qualitative methodology consisting of three phases. The first is a literature review, followed by an indepth analysis of the creation process of Queerskins: Ark through a series of semi-structured interviews with the authors. Finally, the virtual footage is analysed by applying our own model designed in three blocks. The research projects carried out to test the impact of VR audiovisual narrative on the viewer have always opted for perspective-taking strategies. On the other hand, the work under study experiments with the application of expressive and artistic resources for the same purpose. The results demonstrate the existence of alternative strategies to generate empathic responses in the audience.
\end{abstract}

\section{Keywords}

volumetric cinema, presence effect, empathy, virtual reality, audiovisual production

\section{Introducción}

Los modos de expresión artísticos han evolucionado permitiendo la representación real, imaginaria 0 implícita de entornos análogos 0 virtuales y de otras personas, aumentando el poder inmersivo de las experiencias mediáticas (Blascovich et al. 2002, 106). La evolución del medio audiovisual se ha desarrollado en un complejo campo de interconexiones más allá de la genealogía causal (Foucault 1977, 146). En la actualidad, las técnicas del cine volumétrico permiten el registro tridimensional de imágenes en movimiento de actores reales y su implementación en entornos virtuales para la construcción de experiencias fílmicas interactivas. El cine volumétrico no es solo una innovación tecnológica, sino un nuevo medio audiovisual que altera el papel del director y empodera al espectador en el proceso de creación y fruición narrativa (Pietroszek 2020, 1). Se trata de un medio interactivo, participativo y narrativo que enriquece el lenguaje del storytelling audiovisual, y sus películas pueden presentarse en cualquier punto del continuo realidad-virtualidad mediante un head mounted display o un visor de realidad mixta (Pietroszek y Eckhardt 2020, 3).
La convergencia audiovisual, apunta Thomas Elsaesser, versa sobre modelos alternativos de pensamiento entre el cambio y la continuidad, enfocando su mirada hacia el cine primitivo $(2004,80)$. Se pregunta, al mismo tiempo, cómo de defectuosa es la historia del cine, atendiendo a todas aquellas omisiones relativas al primer cine y a los discursos posteriores sobre el color, el sonido o la experimentación con pantallas y sistemas de visión estereoscópicos que paulatinamente se han quedado en el olvido. Hechos de la evolución del séptimo arte que vuelven a tener relevancia en su trazabilidad en relación con el momento presente, donde la irrupción de las tecnologías inmersivas abre de nuevo el debate sobre la confluencia de los medios. En este sentido, la RV «trata de experiencias generadas por ordenadores que simulan señales sensoriales de la realidad física más cercanas a esta que las experiencias generadas utilizando tan solo una pantalla y altavoces» (Wilson, citado en Simó 2019, 133). Partiendo de estas ideas, podemos inferir que el cine de RV atiende un momento análogo al cine de atracciones, «al alentar a los espectadores a sumergirse en la imagen como entorno total en lugar de relacionarse con la pantalla como una ventana al mundo» (Elsaesser 2004, 83). 


\section{artnodes}

https://artnodes.uoc.edu

Estrategias del cine volumétrico para generar empatía en la audiencia. El caso de Queerskins: Ark

La práctica cinematográfica ostenta una función modeladora de los modos de pensamiento (Deleuze 1987), definiendo también cómo el espectador percibe su entorno, además de establecer las relaciones entre lo que se ve y lo que se sabe o conoce, desde la perspectiva de Todorov (1969) y Genette (1983), revisitada por Gaudreault y Jost (1995). De este modo, el cine volumétrico de RV retoma ciertas cuestiones relativas a la presencia, la interfaz, los modelos narrativos, el punto de vista, la dirección de la mirada del espectador, la inmersión y la empatía a través de la suspensión de la incredulidad (Bates 1991; Slater y Wilbur 1997; Green y Brock 2000; Cho et al. 2016; Syrett et al. 2016; Nielsen et al. 2016; Bucher 2017; Mateer 2017; todo ellos citados en Martínez-Cano y Roselló Tormo 2020, 113-114).

El concepto de empatía ha sufrido una constante evolución en su definición desde comienzos del siglo XX, cuando fue incorporado al inglés (empathy) desde el término alemán Einfünlung, una forma de simpatía estética que permitía a la audiencia apreciar los movimientos abstractos de la danza contemporánea (OImedo Carrillo y Montes Berges 2009 , 1). Actualmente su definición gira en torno a la habilidad de sentir, comprender e imaginar el estado emocional del otro, de los demás, y puede ser dividida en tres clases: experiencias de compartir las emociones del otro, un proceso cognitivo de consideración del punto de vista del otro, o como una preocupación prosocial, que induce a la motivación por mejorar el estado del otro (Zaki y Ochsner 2012, 675). Esta clasificación es traducida a tres niveles: empatía cognitiva, convergencia emocional y respuesta empática (Janssen 2012), en el contexto de las tecnologías empáticas y la computación afectiva, áreas que sostienen que la tecnología puede ayudar a identificar déficits de empatía o a entrenar a los individuos en sus respuestas empáticas, resultando en un beneficio en las sociedades contemporáneas, revitalizando los valores empáticos (Janssen 2012 , 114). En la convergencia emocional se incluye la tendencia de imitar $y$ sincronizar automáticamente las expresiones faciales, vocalizaciones, posturas y movimientos con aquellos de otra persona (Hatfield et al. 1994, 5).

Partiendo de estas ideas, la imagen en movimiento ostenta un potencial para la creación de empatía en la audiencia. «La reproducción de las condiciones de percepción del entorno a las que nos expone el consumo de lo audiovisual [...] produce una diferencia sustancial respecto de los otros soportes en la circulación de mensajes» (Pajoni 2008, 432). Esta capacidad modeladora es explicada como la internalización del audiovisual en nuestros modos de cognición y experiencia, lo que se identifica como el «efecto cine» (Elsaesser 2004, 76). La práctica cinematográfica esconde la parafernalia técnica al ojo del espectador para conseguir la inmersión en la diégesis narrativa (Bordwell et al. 1985). Además, en el cine volumétrico se deben ocultar ciertas estrategias creativas que han de estar accesibles para el espectador de manera inconsciente, como en el caso de las técnicas de interpretación de los actores, ya que la percepción cinemática queda asumida en nuestros modos de conocer y experimentar nuestro entorno cotidiano desde nuestra concepción corporal (embodied experience) (Biocca 1997; Gibbs Jr. et al. 2004; Kilteni et al. 2012; Shin 2018).

\subsection{La importancia de la dirección de actores en el cine volumétrico}

El cine convencional establece una frontera infranqueable entre la corporeidad de la pantalla y la del espectador en su posición en el patio de butacas, donde da lugar su experiencia, y esta depende de la conexión de las imágenes en movimiento en cuerpos expresivos y su capacidad de tensión emocional, transmitida desde la expresividad de los cuerpos representados en el campo visual hacia el cuerpo del espectador (Arnheim 1954, 408, citado en D’Aloia 2012, 97). El cine volumétrico elimina esta distancia, acercando la corporalidad de intérpretes y espectadores y propiciando una dinámica de la expresión kinestésica más cercana al teatro, con lo que la ficción es percibida como más auténtica debido al nivel de presencia física que consigue (Popat 2016).

La problemática surge ya con el cine mudo, donde los autores debían encontrar el modo de hacer que la audiencia sintiese la experiencia que se quería transmitir, y para esto era necesario crear un lenguaje corporal legible. El modo de resolver esta necesidad fue la amplificación de los gestos de los actores (Doane 1980, 162). La exageración de los movimientos coreografiados de los intérpretes ejerce de vínculo empático con la audiencia en relación con el storytelling, por lo que, para el espectador, no es necesario entender el medio desde un plano intelectual, necesita sentirlo (Reynolds 2012).

Hasta la irrupción del cine volumétrico, estos aspectos no habían entrado en juego, ya que no se trabajaba con actores reales en las producciones de VRcinema, en las que la imagen sintética 3D era su única configuración posible. La interpretación, gestualidad y movimientos de los actores resultan cruciales en los procesos de configuración de presencia. La audiencia «repite reflexivamente de un modo debilitado todo el sistema de movimientos de un actor: como resultado de los movimientos producidos, las incipientes tensiones musculares del espectador se liberan en la emoción deseada» (Eisenstein 1987, 37). En este sentido, el actor, entendido como «atleta de la interpretación, debe ser capaz de comunicar emociones a través de sus habilidades motoras e inducir estados psicológicos en el espectador a través de sus respuestas motoras» (D'Aloia 2012, 97). La planitud de los cuerpos fílmicos trasciende en su posibilidad de representación volumétrica hacia la presencia virtual fílmica, por tanto, las producciones de ficción RV deben considerar las técnicas de trabajo actoral con el objetivo de potenciar sus estrategias narrativas.

\subsection{Estrategias RV para generar respuestas empáticas}

Las principales líneas de investigación que tratan de medir el impacto de las producciones audiovisuales de RV para generar respuestas 


\section{artnodes}

empáticas se han basado en la estrategia de toma de perspectiva. Trabajos como «1000 Cutjourney» (Bailenson et al. 2018) utilizan esta técnica, en este caso para sumergir al usuario en la experiencia de un hombre negro en diferentes situaciones de racismo. En la misma línea, el proyecto titulado «Building long-term empathy: A large-scale comparison of traditional and virtual reality perspective-taking» (Herrera et al. 2018), en el que han trabajado con la experiencia RV Becoming homeless: A human experience, demuestra que las tareas de toma de perspectiva son efectivas para generar empatía en la audiencia a medio y largo plazo.

Otro proyecto que aborda estas cuestiones, «The effect of embodied experiences on self-other merging attitude and helping behaviour» (Ahn et al. 2013), concluye que la estrategia de solapamiento de uno mismo con el otro en la ficción de RV es transferida al mundo físico y se traduce en la adquisición de valores prosociales por parte de la audiencia. Además, el artículo «Walk a mile in digital shoes: The impact of embodied perspective-taking on the reduction of negative stereotyping immersive in virtual environments» (Yee y Bailenson 2006), remarca el poder de las experiencias virtuales de toma de perspectiva para reducir los estereotipos sociales negativos. El uso único de las estrategias de toma de perspectiva en los proyectos mencionados señala la limitación de recursos creativos, justificándose con ello la exploración de alternativas para la generación de respuestas empáticas.

\section{Metodología}

Este trabajo parte de una metodología cualitativa dividida en tres fases. Una primera de revisión de la literatura, para, posteriormente, Ilevar a cabo una serie de entrevistas semi-estructuradas a los autores de la obra objeto de estudio: la escritora y directora Illya Szilak, el director técnico y creativo Cyril Tsiboulski y el coreógrafo Brandon Powers. Estas entrevistas se han realizado durante el mes de agosto de 2020, previo al estreno de la obra en la sección Venice VR Expanded de la 77 Muestra Internacional de Arte Cinematográfico La Biennale di Venezia 2020, y, posterior a su visionado, durante la primera semana de septiembre de 2020. Por último, se ha desarrollado una herramienta de análisis para el estudio de Queerskins: Ark (Szilak y Tsiboulsk 2018).

Esta herramienta se compone de tres bloques de variables. En el primero se evalúa si la presencia de la obra objeto de estudio puede considerarse como social, ambiental o personal (Heeter 1992, 263-264). En este mismo bloque se evalúan también las reglas de interacción, si existe o no navegación, así como el tipo de movimientos dentro del entorno virtual, según establecen Slater y Wilbur (1997) en relación con la consecución de presencia efectiva. En el segundo se abordan las cuestiones relativas a la realización audiovisual. Finalmente, en el tercero, la estructura narrativa.

\section{Resultados}

Queerskins: Ark es una obra de arte narrativo de RV que presenta actores y bailarines humanos con registro volumétrico 3D. El cine volumétrico de RV es un medio que requiere una nueva forma de pensar la dirección audiovisual, en la que se han de considerar todos y cada uno de los elementos que conforman el discurso y su lenguaje (Bucher 2017). Desde la escritura del guion se deben pensar todas sus capas y fases, incluida la posproducción, que resulta en un proceso intensificado por la alta carga tecnológica de su mixtura con el medio videolúdico, en el que se ha de resolver la tensión generada entre la libertad de acción del espectador y la construcción de su estructura narrativa.

\subsection{Consecución de presencia}

La corporeización (embodiment) y su problemática, en cuanto a su representación y construcción-deconstrucción de identidad conectan con la sensación de presencia (Biocca 1997). La primera y tercera escenas de Queerskins: Ark se han realizado en vídeo 360 sin interacción, siguiendo el criterio representacional del registro fotográfico realista y cumpliendo el principio básico que establece Heeter (1992, 5) en relación con el sentimiento de presencia personal basado en simular nuestra percepción en el mundo real (Lombard y Ditton 1997). Sin embargo, ninguna de las partes del cuerpo del espectador es reconstruida ni visualizada por este en el contexto de la obra objeto de estudio, no se enfunda en ningún tipo de avatar, por lo que no se produce ilusión de transferencia corporal (Slater et al. 2010).

La construcción del espacio fílmico, la puesta en escena, la dirección de actores y el diseño coreográfico constituyen los pilares sobre los que se asienta la consecución de presencia. Todos ellos se han diseñado de forma circular, envolviendo al espectador tanto en las escenas de vídeo 360 como en la de imagen volumétrica. En la escena inicial (figura 1), el personaje principal entra en el campo

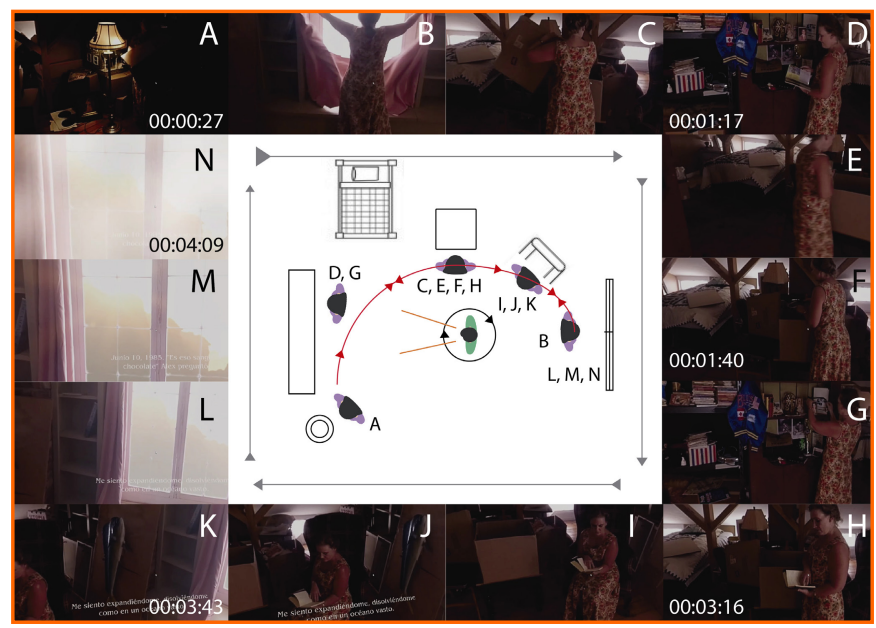

Figura 1. Planta escenográfica y capturas de pantalla del visionado de Queerskins: Ark (Szilak y Tsiboulski, 2020). Fuente: elaboración propia. Intel Studios \& Cloudred@. 


\section{artnodes}

virtual, desarrollando una serie de acciones y movimientos en una trayectoria semicircular alrededor del espectador, desde el que transita al segundo acto: del desván a la playa.

En esta escena de ensueño, los personajes escenifican un baile en torno al espectador, invitándole a interactuar con sus movimientos, con los que modifica inconscientemente el transcurso de la coreografía diseñada para potenciar la sensación de presencia.

«En mi trabajo como coreógrafo en XR estoy realmente interesado en cómo podemos mover no solo a los bailarines en el espacio, sino al usuario con el visor RV situado en el espacio virtual y hacer lo que me gusta llamar el enfoque de adentro hacia fuera, que esencialmente consiste en diseñar la experiencia en torno a los movimientos de cuerpo del espectador, para que se sienta de un modo concreto a la hora de interiorizar el sentimiento que la obra propone» (B. Powers, comunicación personal, 5 de septiembre de 2020).

En este sentido, identificamos la creación de presencia ambiental (Heeter 1992; Slater y Wilbur 1997), tanto por la estrategia de la puesta en escena y coreografía circular como por el modo en que el espacio responde a nuestras intervenciones como público en la escena central de la playa, donde, por ejemplo, la luz se ve alterada dependiendo de nuestros movimientos de brazos, aumentando si los abrimos, en una mímica de los movimientos de apertura de los actores durante su baile, conectando así con el concepto de empatía kinestésica.

Además, para involucrar a la audiencia en la danza de los personajes, uno de los principales desafíos, puesto que se llega a esta desde una escena sin capacidad interactiva, también se aplicó el diseño circular de la mise-en-scène, guiando la mirada del espectador y llamando a su acción, como sucede cuando al input sonoro nos giramos para ver llegar a uno de los personajes acercarse al otro en una trayectoria semicircular, volviendo ambos a tumbarse en la arena mientras mantienen una conversación cuya frase final («touch me») funciona como trigger que da lugar al inicio de la danza (figura 2).

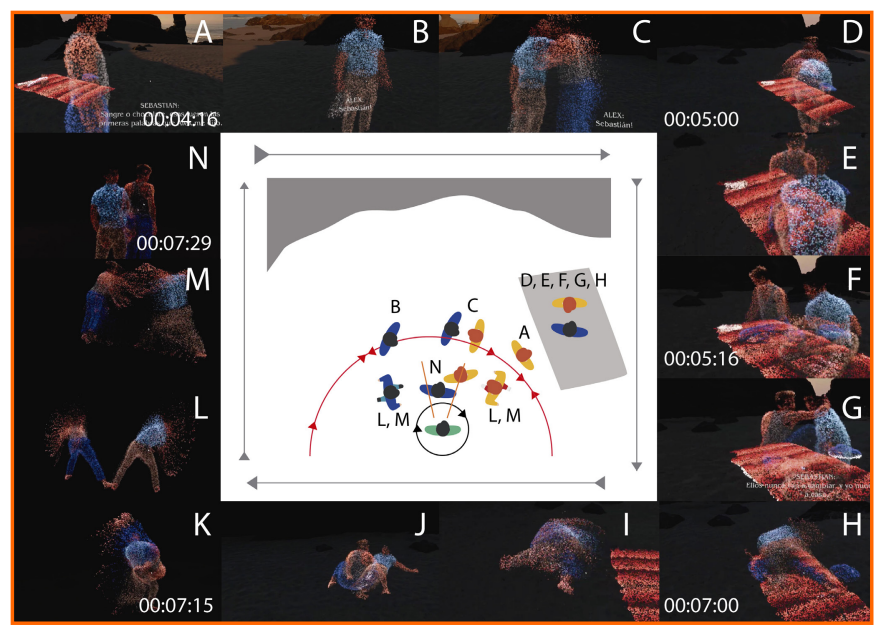

Figura 2. Planta escenográfica y capturas de pantalla del visionado de Queerskins: Ark (Szilak y Tsiboulski, 2020). Fuente: elaboración propia. Intel Studios \& Cloudred@
La voluntad de acción del espectador es incorporada a través de un sistema de interacción sutil, en el que, dependiendo de los movimientos ejercidos en relación con los actores mientras bailan, nos acercamos 0 alejamos, levitamos o descendemos. Este sistema se presenta de un modo intuitivo, el participante debe descubrir durante el transcurso de la secuencia interactiva cómo el entorno virtual y los bailarines responden a sus acciones. Por tanto, el primer factor establecido por Slater y Wilbur (1997) se cumple de manera parcial, pues a pesar de no presentarse esta información de una manera explícita, el sistema es de fácil manejo para el espectador. «Queríamos crear un lenguaje cuerpo a cuerpo. Algún tipo de conexión íntima entre el espectador y los bailarines a un nivel corporal, sin convertirlos en marionetas. Los personajes no son objetos de juego, y como espectador, necesitas sentirte conectado a ellos y sentir que los movimientos de tu cuerpo afectan a lo que está sucediendo» (I. Szilak, comunicación personal, 14 de agosto de 2020).

Sin embargo, la navegación visual del entorno responde a las características de la percepción en un espacio real. Aunque el realismo potencia la sensación de presencia, el criterio representacional fluctúa del realismo a la representación iconográfica en un menor grado durante la escena central, donde los personajes son creados a partir de un sistema de partículas. Al final de esta escena, se pierde la referencia espacial, ubicándonos en un no lugar, en una abstracción donde solo conviven los dos bailarines y el espectador. A pesar de este hecho, la presencia ambiental no se minimiza, sino que da lugar a un tipo de presencia resultado de sentir una conexión con la experiencia íntima de la que se participa, promoviendo de nuevo la empatía del público con respecto a la temática de la obra. La libertad de movimiento de espectador para incorporarse a la danza íntima de los personajes e intervenir en el devenir de la misma dependiendo de sus gestos en el espacio dan lugar a una profunda presencia personal. «En el sentido de que podemos crear presencia, y la presencia está íntimamente relacionada con el movimiento, podemos hacer sentir al espectador como si estuviera en este otro entorno donde tiene esta oportunidad de ser una persona reencarnada virtualmente para compartir una situación íntima de dos hombres que se aman. Queremos que esta experiencia le acompañe» (I. Szilak, comunicación personal, 6 de septiembre de 2020).

\subsection{Dirección y realización audiovisual volumétrica}

La dirección y producción de Queerskins: Ark pone de manifiesto los desafíos de la técnica de registro volumétrico. Rodada en los Estudios Intel, precisó de una solución creativa pues el sistema de cámaras y sensores que circundan el espacio semiesférico de la cúpula del estudio solo permite la captura de treinta segundos de metraje por cada toma, además de requerir un interludio de dos minutos entre tomas para volcar el material grabado y resetear las cámaras y sensores para la siguiente. Por tanto, para la escena central de la danza tuvieron que diseñar una estructura secuencial con el fin de mantener el rácord de movimiento de los actores. 


\section{artnodes}

https://artnodes.uoc.edu

Estrategias del cine volumétrico para generar empatía en la audiencia. El caso de Queerskins: Ark

La dirección de actores también se intensifica, ya que con el fin de generar una respuesta empática a partir de los gestos y movimientos de los actores se implementaron las técnicas del cine mudo, ampliando la gestualidad de los intérpretes en su acting para crear un lenguaje corporal legible, capaz de ser interpretado por la audiencia de un modo natural.

El plano sonoro ha sido diseñado utilizando el sistema binaural para producciones de RV, compuesto principalmente por el sonido diegético de las acciones presentadas en la puesta en escena, con voces en offy diálogos de los actores sobre los paisajes sonoros del espacio ficcional, a lo que se suma una banda sonora en la escena central compuesta por música de piano e instrumentos de cuerda en la que se sustenta el fin lírico de la escena. La producción sonora de la obra también participa en el sistema de interacción, pues guía la mirada del visitante dependiendo del lugar desde el que se escucha el registro de audio.

\subsection{Estructura narrativa}

La estructura se compone de tres actos (véase la figura 3). El primero en el desván, a modo de prólogo que da entrada a la segunda escena, en la que atendemos a la conversación íntima de los dos amantes en la playa el día de su aniversario, tras la que accedemos a la danza ritual de ambos en una abstracción espacial, justificada en la ensoñación del personaje principal de la escena inicial. Al terminar este segundo acto pasamos a la tercera y última escena a modo de epílogo.

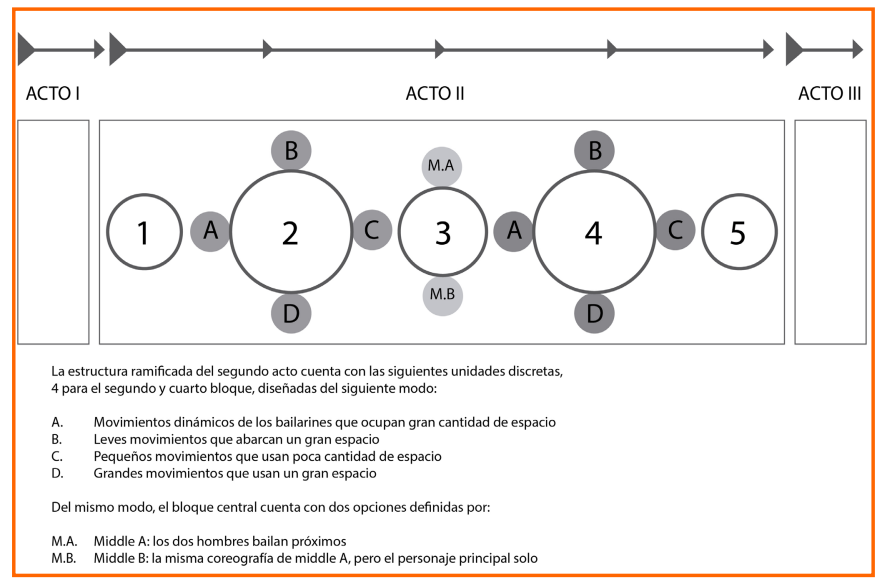

Figura 3. Estructura narrativa de Queerskins: Ark (Szilak y Tsiboulski, 2020). Fuente: elaboración propia.

El segundo acto presenta además una estructura propia más compleja, pues es la que incorpora la capa de interacción por parte del espectador. Su estructura fue diseñada a partir de diferentes segmentos de coreografía relacionados con diferentes momentos de la historia de Sebastian y Alex. Este acto se divide en cinco bloques, el inicial y el final no varían, mientras los tres centrales responden a la interacción del usuario, resultando en un determinado fragmento coreográfico. El segundo y cuarto bloque contienen cuatro opciones diferentes, de las cuales se visualiza una dependiendo de nuestros movimientos en el espacio, mientras el bloque central dispone de dos opciones (véase la figura 3).

\section{Discusión y conclusiones}

El potencial de la RV para promover respuestas empáticas es atribuido a cambios en los procesos emocionales y cognitivos que ocurren cuando los participantes pueden moverse libremente. El movimiento es un medio para la transferencia de conceptos y emociones desde la conciencia de un individuo a la de otro (Corsa 2016). «La función de la danza es expresar sentimientos por medio del movimiento corporal y la capacidad empática del público» (Martin 1965, citado en Corsa 2016, 7). Las experiencias mediadas del audiovisual poseen el potencial de generar empatía, especialmente aquellas con una alta intensificación kinestésica (D’Aloia 2012, 95; Reynolds 2012). Por tanto, resulta coherente la elección de la danza dentro de la estrategia narrativa de Queerskins:Ark, así como la implementación de técnicas de interpretación del cine mudo con el fin de promover la respuesta empática del espectador. En consecuencia, el cine volumétrico de RV no se sustenta por la tecnificación del medio, pues su impacto depende de las estrategias narrativas y creativas por encima de lo tecnológico.

Queerskins: Ark demuestra la viabilidad de otras estrategias para promover la empatía en el espectador más allá de la estrategia de toma de perspectiva, y en ella se comprueba la eficacia de la kinestesia con este objetivo. De este modo, la dirección de cine volumétrico de RV no se centra tanto en las cuestiones relacionadas con la posición de la cámara, sino en la coreografía de los actores. Se identifica la efectividad del uso del círculo para diseñar una puesta en escena que sitúa al espectador en el espacio ficcional, instándole a recorrer visualmente el entorno diegético. Se consigue así aumentar la convergencia emocional y potenciar la sensación de presencia para generar respuestas empáticas.

Podemos concluir que Queerskins: Arkes un paradigma del uso de Ios recursos expresivos y artísticos para construir historias inclusivas. La obra sitúa a la audiencia en situaciones íntimas a las que no tendría acceso de otro modo, tratando de obtener una respuesta empática desde la propia mirada del espectador en relación con la realidad que presencia, sin solapar la identidad del participante con la de los personajes de la ficción.

La RV es un medio espacial y en él sentimos los cuerpos en el espacio de un modo diferente a como lo hacemos frente a la pantalla, pues nos sitúa dentro del espacio-tiempo ficcional. En este sentido, los autores apuestan por la creación de un lenguaje gestual que conecta con la empatía kinestésica. Esta estrategia potencia el embodiment 


\section{artnodes}

https://artnodes.uoc.edu

de actores y espectadores en el entorno virtual, materializando la corporeidad de la experiencia y amplificando el factor emocional de su discurso.

\section{Referencias bibliográficas}

Ahn, Sun Joo, Amanda Minh Tran Le y Jeremy Bailenson. «The effect of embodied experiences on self-other merging, attitude, and helping behavior». Media Psychology, vol. 16, no. 1 (2013): 7-38. DOI: https://doi.org/10.1080/15213269.2012.755877.

Biocca, Frank. «The cyborg's dilemma: progressive embodiment in virtual environments». Journal of computer-mediated communication, vol. 3, no. 2 (1997): JCMC324. DOI: https://doi. org/10.1111/j.1083-6101.1997.tb00070.x.

Blascovich, Jim, Jack Loomis, Andrew C. Beall, Kimberly R. Swinth, Crystal L. Hoyt y Jeremy N. Bailenson. «Immersive virtual environment technology as a methodological tool for social psychology». Psychological Inquiry, vol. 13, no. 2 (2002): 103-124. https://www. researchgate.net/publication/2540081_Immersive_Virtual_Environment_Technology_as_a_Methodological_Tool_for_Social_ Psychology. D0l: https://doi.org/10.1207/S15327965PLI1302_01

Bordwell, David, Janet Staiger y Kristin Thompson. The classical Hollywood cinema: Film style \& mode of production to 1960. Nueva York: Columbia University Press, 1985. D0I: https://doi. org/10.2307/1772109

Bucher, John. Storytelling for virtual reality: methods and principles for crafting immersive narratives. Taylor \& Francis, 2017. D0l: https://doi.org/10.4324/9781315210308

Cogburn, Courtney D., Jeremy Bailenson, Elise Ogle, Tobin Asher, and Teff Nichols. «1000 Cut Journey». En ACM SIGGRAPH, 2018. DOI: https://doi.org/10.1145/3226552.3226575

Corsa, Andrew J. «Kinesthetic empathy, dance, and technology». Polymath. An Inter-disciplinary Arts and Sciences Journal, vol. 6 , no. 2 (2016): 1-34. https://ojcs.siue.edu/ojs/index.php/polymath/ article/view/3140.

D’Aloia, Adriano. «Cinematic empathies. Spectator involvement in the film experience» (2011). En Kinesthetic empathy in creative and cultural practices. Intellect Ltd., 2012.

Deleuze, G. La imagen-tiempo: Estudios sobre cine, vol. 2. Barcelona: Paidós, 1987.

Doane, Mary Ann. «The Voice in the Cinema: The Articulation of Body and Space». Yale French Studies, no. 60 (1980): 33-50. DOl: https://doi.org/10.2307/2930003

Eisenstein, S., S. M. Ejzenštejn, H. Marshall y Press Cambridge University. Nonindifferent nature: Film and the structure of things. Nueva York: Cambridge University Press, 1987.

Elsaesser, Thomas. «The new film history as media archaeology». Cinémas. Revue d'études cinématographiques/Cinémas. Journal of Film Studies, vol. 14, no. 2-3 (2004): 75-117. D0l: https://doi. org/10.7202/026005ar

Foucault, Michel. Language, counter-memory, practice: Selected essays and interviews. Cornell University Press, 1977.

Gaudreault, André y François Jost. El relato cinematográfico. Buenos Aires: Paidós, 1995.

Genette, Gérard. Narrative discourse: An essay in method, vol. 3. Cornell University Press, 1983.

Gibbs Jr, Raymond W., Paula Lenz Costa Lima y Edson Francozo. "Metaphor is grounded in embodied experience». Journal of Pragmatics, vol. 36, no. 7 (2004): 1.189-1.210. D0l: https://doi. org/10.1016/j.pragma.2003.10.009.

Hatfield, Elaine, John T. Cacioppo y Richard L. Rapson. Emotional contagion: Studies in emotion and social interaction. Cambridge: Cambridge University Press, 1994.

Heeter, Carrie. «Being there: The subjective experience of presence». Presence: Tele-operators \& Virtual Environments, vol. 1, no. 2 (1992): 262-271. DOl: https://doi.org/10.1162/pres.1992.1.2.262

Herrera, Fernanda, Jeremy Bailenson, Erika Weisz, Elise Ogle y Jamil Zaki. «Building long-term empathy: A large-scale comparison of traditional and virtual reality perspective-taking». PloS one, vol. 13, no. 10 (2018): e0204494. D0I: https://doi.org/10.1371/journal. pone.0204494.

Janssen, Joris $H$. «A three-component framework for empathic technologies to augment human interaction». Journal on Multimodal User Interfaces, vol. 6, no. 3 (2012): 143-161. https://link. springer.com/article/10.1007/s12193-012-0097-5. D0l: https:// doi.org/10.1007/s12193-012-0097-5

Kilteni, Konstantina, Raphaela Groten y Mel Slater. «The sense of embodiment in virtual reality». Presence. Teleoperators and Virtual Environments, vol. 21, no. 4 (2012): 373-387. D0I: https://doi. org/10.1162/PRES_a_00124

Lombard, Matthew y Theresa Ditton. «At the heart of it all: The concept of presence». Journal of computer-mediated communication, vol. 3, no. 2 (1997): JCMC321. D0I: https://doi. org/10.1111/j.1083-6101.1997.tb00072.x.

Martínez-Cano, Francisco Julián y Emilio Roselló Tormo. «La dirección y realización audiovisual de realidad virtual. Análisis de Queerskins: A Love Story. Una aproximación al cine volumétrico» ASRI: Arte y sociedad. Revista de investigación, no. 18 (2020): 111-125. https://dialnet.unirioja.es/servlet/articulo?codigo=7846298.

Olmedo Carrillo, Pablo y Beatriz Montes Berges. «Evolución conceptual de la empatía». Iniciación a la Investigación, no. 4 (2009). https://revistaselectronicas.ujaen.es/index.php/ininv/article/ view/307.

Pajoni, Hernán. «Lo audiovisual: realidad o experiencia vicaria». En Artes Y Medios Audiovisual: Un Estado De Situación li: Las Prácticas Mediáticas Pre Digitales Y Post Analógicas, editado por Jorge La Ferla, 432-440. Buenos Aires: Aurelia Rivera/Nueva Librería, 2008. 


\section{artnodes}

Pietroszek, Krzysztof. «Volumetric filmmaking». Encyclopedia of Computer Graphics and Games. En Newton Lee (ed.). Springer International Publishing (2020): 1-3. https://doi.org/ 10.1007 / 978-3-319-08234-9_391-1.

Pietroszek, Krzysztof y Christian Eckhardt. «Volumetric capture for narrative films». Actas del 26. ${ }^{\circ} \mathrm{ACM}$ Symposium on Virtual Reality Software and Technology, no. 28 (2020). DOI: https://doi. org/10.1145/3399715.3399957.

Popat, Sita. «Missing in action: Embodied experience and virtual reality». Theatre Journal, vol. 68, no. 3 (2016): 357-378. D0I: https://doi.org/10.1353/ti.2016.0071

Reynolds, Dee. «Kinesthetic empathy and the dance's body: From emotion to affect». En Kinesthetic empathy in creative and cultural practices. Intellect Ltd., 2012.

Shin, Donghee. «Empathy and embodied experience in virtual environment: To what extent can virtual reality stimulate empathy and embodied experience?». Computers in Human Behavior, no. 78 (2018): 64-73. https://doi.org/10.1016/j.chb.2017.09.012.

Simó, Águeda. «La realidad virtual en la creación artística: conceptos, tecnologías, trayectoria y actualidad». Arte y Políticas de Identidad, no. 20 (2019): 131-146. D0I: https://doi.org/10.6018/ reapi.389521.
Slater, Mel, Bernhard Spanlang, Maria V. Sanchez-Vives y Olaf Blanke. «First person experience of body transfer in virtual reality». PloS one, vol. 5, no. 5 (2010): e10564. D0l: https://doi.org/10.1371/ journal.pone. 0010564 .

Slater, Mel. «Measuring presence:A response to the witmer and singer presence questionnaire». Presence, vol. 8, no. 5 (1999): 560-565. D0l: https://doi.org/10.1162/105474699566477

Slater, Mel y Sylvia Wilbur. «A framework for immersive virtual environments (Five): Speculations on the role of presence in virtual environments». Presence. Teleoperators \& Virtual Environments, vol. 6, no. 6 (1997): 603-616. Dol: https://doi.org/10.1162/ pres.1997.6.6.603

Todorov, Tzvetan. «Structural analysis of narrative». Artículo presentado en el «Novel: a forum on fiction», 1969. D0l: https://doi. org/10.2307/1345003

Yee, Nick y Jeremy N. Bailenson. «Walk a mile in digital shoes: The impact of embodied perspective-Taking on the reduction of negative stereotyping in immersive virtual environments». Proceedings of PRESENCE, no. 24 (2006): 26. https://vhil.stanford.edu/mm/2006/ yee-digital-shoes.pdf.

Zaki, Jamil y Kevin N. Ochsner. «The neuroscience of empathy: Progress, pitfalls and promise». Nature Neuroscience, vol. 15, no. 5 (2012): 675-680. DOl: https://doi.org/10.1038/nn.3085 


\section{artnodes}

https://artnodes.uoc.edu

Estrategias del cine volumétrico para generar empatía en la audiencia. El caso de Queerskins: Ark

\section{CV}

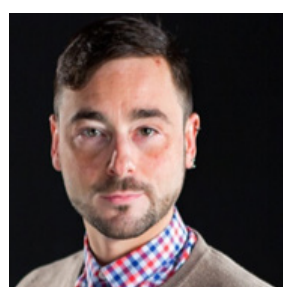

Francisco-Julián Martínez-Cano

Universidad Miguel Hernández

francisco.martinezc@umh.es

Francisco-Julián Martínez-Cano (1983) es doctor en Bellas Artes y profesor ayudante doctor de la Universidad Miguel Hernández (UMH). Desde 2006, su trayectoria artística se centra en la mezcla de ciencia y arte a fin de generar nuevos discursos visuales. Como investigador, estudia y analiza las tecnologías de realidad virtual y realidad aumentada y su hibridación con cine y videojuegos, para la creación de nuevos productos de entretenimiento audiovisuales, prestando atención a los fenómenos de transmediatización contemporáneos y los mass media. Ha realizado exposiciones nacionales e internacionales, de las que destacan «Condición de posibilidad» (Palacio de los Condes de Gabia de Granada, 2005); Ia IX Exposición Audiovisual UPV-BBVA (Bilbao, 2006), y «Gabinete de papeles» (Museo de Arte Contemporáneo de Elche, 2011). Asimismo, cabe remarcar el proyecto «Terrorismo de masas» (Centro 14 de Alicante, 2014) y su participación en el Miami New Media Festival 2019, en la Miami Art Week 2019 y en el Miden Video Art 2020 con la obra audiovisual ALC-SKG. Es miembro del grupo de investigación OCAP, cuyos objetivos son el estudio de la imagen audiovisual y las funciones comunicativas de los medios emergentes. Ha sido profesor invitado en la Universidad de Beira Interior (Portugal), en la Universidad de Évora (Portugal), en el Plymouth College of Art (Plymouth, Reino Unido) y en la Universidad de las Californias Internacional (Tijuana, México). 


\section{artnodes}

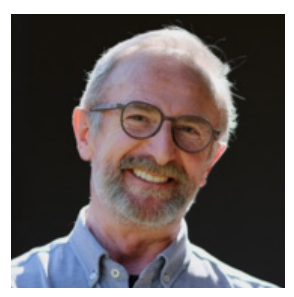

\section{Emilio Roselló-Tormo}

Universidad Miguel Hernández

e.rosello@umh.es

Emilio Roselló-Tormo es doctor en Bellas Artes y profesor titular de la Universidad Miguel Hernández (UMH). Estudió en la Universidad Politécnica de Valencia, donde se doctora en Bellas Artes (1994) con una investigación sobre animación de la imagen virtual. Su trayectoria investigadora se desarrolla como actividad artística a partir de la década de los noventa a través de la conceptualización de la imagen, la representación y la comunicación. Desde la electrografía, la fotografía digital y la imagen de síntesis, ha elaborado en la praxis diferentes discursos conceptuales y narrativos acerca de la imagen como pensamiento. En la última década se ha centrado más en la fotografía conceptual; es decir, en el hecho de que la fotografía reflexiona sobre sí misma y sobre el hecho fotográfico desde el lenguaje específico de la denominada era posfotográfica. Desde 2001, hasta la actualidad, es el IP del grupo de investigación OCAP, espacio-lugar para la observación multilateral que centra su interés en el estudio de la imagen visual y audiovisual, y en la exploración de las funciones comunicativas que Ios nuevos medios emergentes aplican con versatilidad. Es miembro del colectivo internacional Contemporáneos, en cuyo contexto desarrolla proyectos fotográficos independientes y series colectivas. Fue investigador principal del proyecto «Mapa de la memoria», financiado por la Fundación Víctimas del Terrorismo, la Diputación de Alicante, el Instituto Alicantino de Cultura Juan Gil-Albert y la Caja de Ahorros del Mediterráneo (CAM, Obra Social); e IP del Laboratorio de Ideas, Diseño e Innovación IDI_Lab, financiado por el programa de investigación de Bancaja.

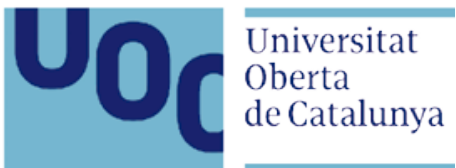

